Claremont Colleges

Scholarship@ Claremont

All HMC Faculty Publications and Research

HMC Faculty Scholarship

$1-1-2004$

\title{
Semilinear Equations with Discrete Spectrum
}

Alfonso Castro

Harvey Mudd College

\section{Recommended Citation}

Castro, Alfonso. "Semilinear equations with discrete spectrum”, Contemporary Mathematics, 357 (2004), pp. 1-16.

This Article is brought to you for free and open access by the HMC Faculty Scholarship at Scholarship @ Claremont. It has been accepted for inclusion in All HMC Faculty Publications and Research by an authorized administrator of Scholarship @ Claremont. For more information, please contact scholarship@cuc.claremont.edu. 


\title{
Semilinear equations with discrete spectrum
}

\author{
Alfonso Castro
}

\begin{abstract}
This is an overview of the solvability of semilinear equations where the linear part has discrete spectrum. Semilinear elliptic and hyperbolic equations, as well as Hammerstein integral equations, are used as motivating examples. The presentation is intended to be accessible to non experts.
\end{abstract}

\section{INTRODUCTION}

These lectures deal with the study of equations of the type

$$
L(u)=N(u),
$$

where $L$ is linear operator with discrete spectrum $\sigma(L)=\left\{\lambda_{i} ; i=1,2, \ldots\right\}$. We recall that if $L$ is a linear operator defined on a dense subset of a Hilbert space then $\sigma(L)$ is the complement in the complex plane of the resolvent $\rho(L)=\{\lambda ; L-\lambda I$ has a bounded inverse $\}$.

A main model for equation (1) is the elliptic problem

$$
\left\{\begin{array}{rlrl}
-\Delta u & =f(x, u) & & x \in \Omega \\
u(x) & =0 & x \in \partial \Omega,
\end{array}\right.
$$

where $\Omega$ is a bounded region in $\mathbf{R}^{n}$ with smooth boundary, $\Delta$ is the Laplace operator, and $f: \Omega \times \mathbf{R} \rightarrow \mathbf{R}$ is a function of class $C^{1}$.

We will see how the interaction of the growth of $N$, generally determined by the numerical range of $N^{\prime}$, with the spectrum of $L$ provides information on the existence, multiplicity, stability, and other properties of the solutions to (1). Such interaction introduces terms such as resonance, jumping nonlinearities, superlinearities, asymptotic nonlinearities, critical growth, and others.

1991 Mathematics Subject Classification. Primary 34B15; Secondary 35J65.

Key words and phrases. Neumann problem, sign-changing solution, nodal region, tilable region. 
While these lectures emphasize the variational case, hence $\sigma(L) \subset \mathbf{R}$, digressions will be made to consider the case where $L$ is a nonselfadjoint perturbation of a selfadjoint operator. This area is yet to be adequately explored.

\section{THE SPECTRUM OF SOME LINEAR OPERATORS}

Let us begin by considering the spectrum of the second order derivative operator, that is,

$$
L(u) \equiv-u^{\prime \prime}=\lambda u, \quad B u=0,
$$

consult [29] ing eigenfunc property

$$
\lambda_{1}
$$

where $B(u)$ is a boundary condition such as $u(0)=u(\pi)=0$ (Dirichlet boundary condition) or $u(t)=u(t+2 \pi)$ (periodic boundary condition). In the case of the Dirichlet boundary condition $\sigma(L)=\left\{j^{2} ; j=1,2, \ldots\right\}$, where as in the case of periodic boundary condition $\sigma(L)=\left\{j^{2} ; j=0,1, \ldots\right\}$, a minor difference. However when we consider a first order perturbation of the second derivative, for example

$$
L(u) \equiv-u^{\prime \prime}+a u^{\prime}=\lambda u, \quad B u=0,
$$

the spectrum changes radically from one boundary condition to the other. For the Dirichlet boundary condition, $\sigma$ is, as before, an increasing sequence of positive real numbers. Namely, $\sigma(L)=\left\{j^{2}-\left(a^{2} / 4\right) ; j=1,2, \ldots\right\}$. The corresponding eigenfunctions are multiples of $u(t)=e^{a t / 2} \sin (j t)$. On the other hand for the periodic boundary condition, $\sigma(L)$ contains only one real number $\lambda=0$. This has a semilinear analog. Namely, while the periodic problem

$$
-u^{\prime \prime}+a u^{\prime}=u^{3}, \quad u(t)=u(t+2 \pi)
$$

is satisfied only by $u \equiv 0$, the equation

$$
-u^{\prime \prime}+a u^{\prime}=u^{3}, \quad u(0)=u(\pi)=0
$$

has infinitely many solutions. More precisely, for each nonnegative integer $j$ the equation (6) has a solution with exactly $j$ interior zeroes.

If $L=-\Delta$ is applied to functions defined on the smooth bounded region satisfying one of the following boundary conditions

$$
\begin{aligned}
u & =0 \text { on } \partial \Omega \text { (Dirichlet), } \\
\frac{\partial u}{\partial \eta} & =0 \text { on } \partial \Omega \text { (Neumann), or } \\
\frac{\partial u}{\partial \eta}+b(x) u & =0 \text { on } \partial \Omega \text { (mixed) }
\end{aligned}
$$

then

$$
\sigma(L)=\left\{\lambda_{1}, \lambda_{2}, \ldots, \lambda_{k}, \ldots\right\}, \quad \lambda_{k} \rightarrow \infty .
$$

Except for the case when $\Omega$ is an interval in $\mathbf{R}$, the eigenvalues need not be simple. However, all the eigenvalues have finite multiplicity. This implies that if $c \notin \sigma(L)$ then the inverse of $L-c I$ is compact. The reader is referred to $[29]$ for a detailed analysis of this topic. In particular, the reader should

where $H$ is $\mathrm{tl}$ functions hav correspondinধ of eigenfuncti

LEMMA 2 $\lambda_{1}$ then $\phi_{1} d$ sponding to $t$ $\left\{x \in \Omega ; \phi_{2}(x)\right.$ [39]).

The proot

When $L \mathrm{i}$ on functions c condition give

$$
u(0, t)=
$$

the spectrum

Unlike the ell both to $+\infty$ ह with $n$ a posil verify, for exa multiplicity, e: the form $\sin (k$ corresponding infinite multif invertible but of $2 \pi$ this set c number theor! 
lence $\sigma(L) \subset \mathbf{R}$, a nonselfadjoint o be adequately

\section{ERATORS}

order derivative

$=0$ (Dirichlet ry condition). In $=1,2, \ldots\}$, where $; j=0,1, \ldots\}, \mathrm{a}$ perturbation of

ion to the other. reasing sequence $=1,2, \ldots\}$. The $2 \sin (j t)$. On the ains only one real hile the periodic

nnegative integer roes.

$\mathrm{h}$ bounded region

alues need not be city. This implies reader is referred the reader should consult [29] for the proof that the eigenvalues $\left\{\lambda_{k}\right\}$ and their corresponding eigenfunctions $\left\{\phi_{k}\right\}$ may be characterized by the following variational property

$$
\begin{aligned}
\lambda_{1}= & \min \left\{\frac{\int_{\Omega}\|\nabla u\|^{2} d x}{\int_{\Omega}\|u\|^{2} d x} ; u \in H-\{0\}\right\}, \text { and for } k>1 \\
\lambda_{k+1}=\min & \left\{\frac{\int_{\Omega}\|\nabla u\|^{2} d x}{\int_{\Omega}\|u\|^{2} d x} ; u \in H-\{0\},\right. \\
& \left.\int_{\Omega} u \phi_{i} d x=0 \text { for } i=1, \ldots, k\right\},
\end{aligned}
$$

where $H$ is the subspace of the Sobolev space (see [1]) of square integrable functions having first order partial derivatives in $L^{2}(\Omega)$ and that satisfy the corresponding boundary condition in (7). From (9) the following properties of eigenfunctions follow.

LEMMA 2.1. If $\phi_{1}$ is an eigenfunction corresponding to the eigenvalue $\lambda_{1}$ then $\phi_{1}$ does not change sign in $\Omega$. If $\phi_{2}$ is an eigenfunction corresponding to the eigenvalue $\lambda_{2}$ then $\phi_{2}$ changes sign exactly once, that is, $\left\{x \in \Omega ; \phi_{2}(x)>0\right\}$ is connected and $\left\{x \in \Omega ; \phi_{2}(x)<0\right\}$ is connected. [39]).

The proof of Lemma 2.1 uses strongly that if $u \in H$ then $|u| \in H$ (see

When $L$ is given by the wave operator $L(u) \equiv \square(u)=u_{t t}-u_{x x}$ acting on functions defined on $[0, \pi] \times \mathbf{R}$ satisfying the Dirichlet-periodic boundary condition given by

$$
u(0, t)=u(\pi, t)=0, u(x, t)=u(x, t+T), \text { for all } x \in[0, \pi], t \in \mathbf{R},
$$

the spectrum of $L$ is the closure of

$$
\left\{k^{2}-\frac{4 \pi^{2}}{T^{2}} ; k=1,2, \ldots, j=0,1, \ldots\right\} .
$$

Unlike the elliptic case $(L=-\Delta)$, now the spectrum of $L$ accumulates both to $+\infty$ and $-\infty$. If $T$ is a rational multiple of $\pi$ or of the form $\sqrt{n} \pi$, with $n$ a positive integer, then $\sigma(L)$ is a discrete subset of $\mathbf{R}$. It is easy to verify, for example, that when $T=2 \pi$ that all the eigenvalues are of finite multiplicity, except for 0 which has infinite multiplicity. In fact functions of the form $\sin (k x) \sin (k t), k=1,2, \ldots$ are mutually orthogonal eigenfunctions corresponding to the eigenvalue 0 . When $T=\sqrt{n} \pi$ all eigenvalues have infinite multiplicity (see [10]). This implies that if $c \notin \sigma(L)$ then $L-c I$ is invertible but $(L-c I)^{-1}$ is not compact. When $T$ is not a rational multiple of $2 \pi$ this set can be very complicated. Its study requires delicate tools from number theory (see $[46]$ ). 


\section{Critical point theory}

Let $H=H_{0}^{1,2}(\Omega)$ be the Sobolev space of square integrable functions with first order partial derivatives in $L^{2}(\Omega)$ and vanishing on $\partial \Omega$ (see [1]). Finding solutions to (2) is equivalent to finding critical points of the functional of the functional $J: H \rightarrow \mathbf{R}$ defined by

$$
J(u)=\int_{\Omega}\left\{\frac{1}{2}|\nabla u|^{2}-F(x, u)\right\} d x,
$$

where $F(x, u)=\int_{0}^{u} f(x, s) d s$. The gradient and Hessian of $J$ are given by

$$
\langle\nabla J(u), v\rangle=\int_{\Omega}\{\nabla u \cdot \nabla v-f(x, u) v\} d x
$$

and

$$
\left\langle D^{2} J(u) v, w\right\rangle=\int_{\Omega}\left\{\nabla v \cdot \nabla w-\frac{\partial f}{\partial u}(x, u) v w\right\} d x .
$$

If $u$ is a critical point of $J$, we define its the Morse index to be the dimension of the largest subspace in which $D^{2} J(u)$ is negative definite. Also for future reference we note that if $\left\{\phi_{1}, \phi_{2}, \ldots,\right\}$ is a set of eigenfunctions corresponding to the eigenvalues given in (8), $X=\operatorname{span}\left\{\phi_{1}, \ldots \phi_{k}\right\}$, and $Y=\operatorname{span}\left\{\phi_{k+1}, \ldots\right\}$ then from $(9)$

$$
\begin{aligned}
\lambda_{k} \int_{\Omega} u^{2}(x) d x & \geq \int_{\Omega}\|\nabla u(x)\|^{2} d x \text { for } u \in X \\
\lambda_{k+1} \int_{\Omega} u^{2}(x) d x & \leq \int_{\Omega}\|\nabla u(x)\|^{2} d x \text { for } u \in Y,
\end{aligned}
$$

the latter are known as Poincaré's inequalities.

The solutions to the equation

$$
\left\{\begin{array}{c}
\square(u)+f(x, t, u)=0 \quad(x, t) \in(0, \pi) \times \mathbf{R} \\
u(0, t)=u(\pi, t)=0, \quad u(x, t)=u(x, t+T), \text { for all } x \in[0, \pi], t \in \mathbf{R}
\end{array}\right.
$$

may also be studied as critical points of a functional. In this case $H$ is given by the space of functions $u:[0, \pi] \times \mathbf{R} \rightarrow \mathbf{R}$ such that $u(0, t)=u(\pi, t)=0$ for all $t \in \mathbf{R}, u(x, t)=u(x, t+T)$ for all $(x, t) \in(0, \pi) \times \mathbf{R}, u, u_{x}, u_{t} \in$ $L^{2}((0, \pi) \times[0, T])$. The functional whose critical points are the solutions of (16) is given by

$$
W(u)=\int_{\Omega}\left\{\frac{1}{2}\left(u_{t}^{2}-u_{x}^{2}\right)-F(x, t, u)\right\} d x,
$$

where $\Omega=(0, \pi) \times[0, T]$, and $F(x, t, u)=\int_{0}^{u} f(x, t, s) d s$. Results for $J$ do not immediately transfer to $W$. A key difference between $J$ and $W$ is that while the integrand in $J$ depending on the partial derivatives of $u$ is positive definite, the one in $W$ is positive definite in an infinite dimensional subspace, negative definite in an infinite dimensional subspace, and may be identically equal to zero in an infinite dimensional subspace. This creates many difficulties when applying arguments based on compactness. 
Another class of semilinear equations that may be studied via critical rable functions on $\partial \Omega$ (see $[1])$. nts of the funcpoint theory are Hammerstein Integral Equations. These are equations of the form

$$
u=\psi(x)+\int_{\Omega} K(x, y) f(x, u(x)) d x,
$$

where $\Omega \subset \mathbf{R}^{N}$ is a bounded region, $f$ is as above, and $K \in L^{2}(\Omega \times \Omega)$ with $K(x, y)=K(y, x)$ for all $x, y \in \Omega$. Since the operator $\mathcal{K}(u)(y) \equiv$ $\int_{\Omega} K(x, y) u(x) d x$ defines a self-adjoint compact operator in $L^{2}(\Omega)$, there exist closed subspaces $X, Y \subset L^{2}(\Omega)$ such that $X \oplus Y=L^{2}(\Omega), \mathcal{K}(X) \subset X$, $\mathcal{K}(Y) \subset Y,\langle\mathcal{K}(u), u\rangle \geq 0$ for $u \in X$, and $\langle\mathcal{K}(v), v\rangle \leq 0$ for $v \in Y$. Assuming further that $\mathcal{K}$ is positive definite in $X$ and negative definite on $Y$ one can find self-adjoint positive definite operators $K_{1}: X \rightarrow X$, and $K_{2}: Y \rightarrow Y$ such that $K_{1}\left(K_{1}(u)\right)=\mathcal{K}(u)$ for all $u \in X$, and $K_{2}\left(K_{2}(v)\right)=-\mathcal{K}(v)$ for all $v \in Y$. Let $F$ be as in (12). Now the functional $I: X \oplus Y=L^{2}(\Omega) \rightarrow R$ defined by

$$
I(u+v)=\int_{\Omega}\left\{\frac{1}{2}\left(u^{2}+v^{2}\right)-F\left(x, K_{1} u-K_{2} v\right)-\psi(x)(u+v)\right\} d x,
$$

is such that $u+v$ is a critical point of $I$ if and only if $K_{1}-K_{2} v$ is a solution to $(18)$ (see $[11]$.)

Hamiltonian systems provide also an important class of semilinear problems with discrete spectrum whose solutions are critical points (see [53].)

\section{Equations without resonance}

Assuming that $\rho(L)$ contains an interval $[\mathrm{a}, \mathrm{b}]$ with $a<b$, we can give a condition on $N$ (from (1)) that guarantees the existence and uniqueness of solution to (1). In fact we have the following result.

Theorem 4.1. Let $H$ be real Hilbert space, and $N: H \rightarrow H$. Suppose that $a<b$ are real numbers and that $[a, b] \subset \mathbf{R}-\sigma(L)$. For each $c \in(a, b)$, there exist $d \in(0, \infty)$ such that if $\|N(u)-c u-(N(v)-c v)\| \leq d\|u-v\|$ for all $u, v \in H$, then the equation (1) has a unique solution. Moreover if $L$ is selfadjoint then $d$ may be any number in $(0, \min \{c-a, b-c\})$.

The proof of this theorem follows from the contraction mapping principle. This is because the equation (1) is equivalent to the fixed point equation

$$
u=(L-c I)^{-1}(N(u)-c u) .
$$

When $(L-c I)^{-1}$ is compact, by appealing to the Schauder fixed point theorem (see [26]), we can further relax the hypotheses on $N$ in the previous theorem. For example, the following holds.

TheOREM 4.2. Let $H$ be real Hilbert space. Suppose that $a<b$ are real numbers and that $[a, b] \subset \mathbf{R}-\sigma(L)$. If there exist $c \in(a, b)$ and $M \in \mathbf{R}$ such that $\|N(u)-c u\| \leq M$ for all $u \in H$, and $(L-c I)^{-1}$ is compact then (1) has a solution. This solution may not be unique (see Theorem 4.5 below). 
From the properties of the properties of the spectrum of $-\Delta$ or the wave operator $\square$ stated in the previous section, it follows that Theorem 4.1 applies to (2) and to (16). In fact the reader is invited to verify the following.

THEOREM 4.3. Let $L$ be the linear operator defined in (2) or (16). If $f$ is continuous function, and $(\partial f / \partial u)(y, u) \in(a, b)$ for all $(y, u)$, and $[a, b] \cap$ $\sigma(L)=\emptyset$, then the corresponding problem has a unique solution.

While Theorem 4.2 applies to (2), it does not apply to (16). In fact, as noted in previous section the inverse of the linear operator defined by $-\Delta+c I$ subject to the boundary conditions in (2) is compact while that corresponding to $\square-c I$, with the boundary condition in (16), is not so. Applying Theorem 4.2 to (2) the following may be established.

THEOREM 4.4. Let $f(x, u)=c u+h(x, u)$ be continuous with $\lim _{|u| \rightarrow \infty} h(x, u)$ $=0$, uniformly for $x \in \Omega$. If $c \in(a, b)$, and $[a, b] \cap \sigma(L)=\emptyset$, then the problem (2) has a solution.

In $[22]$ and [10] sufficient conditions for the existence of solutions to (16) under assumptions related to those Theorem 4.4. See also [58].

Unlike Theorem 4.3, the solution provided in Theorem 4.4 need not be unique. In fact the issue of how many solutions (2) has under the hypotheses even stronger than those in Theorem 4.4 is a matter of many open questions. In fact, let us now assume that $f(x, u)=g(u)$ with

$$
g(0)=0, g^{\prime}(0) \in\left(\lambda_{k}, \lambda_{k+1}\right), \text { and } \lim _{u \rightarrow+\infty} g^{\prime}(u), \lim _{u \rightarrow-\infty} g^{\prime}(u) \in\left(\lambda_{j}, \lambda_{j+1}\right) \text {, }
$$

where the $\lambda_{i}^{\prime} s$ are as in (8) and $\lambda_{0}=-\infty$. When $\Omega$ is an interval in $\mathbf{R}$, it can be shown that (21) implies that (2) has at least $2|k-j|+1$ solutions. This, however, does not extend to higher dimensions. In [31], N. Dancer provided an example of where $\Omega$ is the unit ball in $\mathbf{R}^{N},|k-j|=N-1$ and yet (2) has only three solutions. In [44] it is shown that if $k \neq j$ then (2) has at least two solutions. Also, building on the results of $[\mathbf{2 1}],[\mathbf{1 6}]$, and [13] the following theorem was established in [15].

THEOREM 4.5. Suppose (21) holds with $k=1$ and $j \geq 2$, and that $r g^{\prime}(r)>0$ for $r \in \mathbf{R}-\{0\}$. If there exists $a<\lambda_{j+1}$ such that $g^{\prime}(r) \leq a$ for all $r \in \mathbf{R}$, then (2) has at least five solutions. Two of these solutions change sign.

As stated in section 2, the solutions to

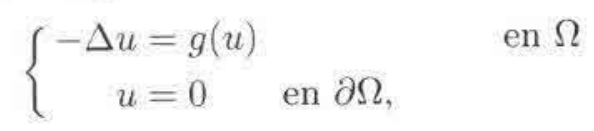

are given by the critical points of

$$
J(u)=\int_{\Omega}\left\{\frac{1}{2}|\nabla u|^{2}-G(u)\right\} d x,
$$

where $G(t)=\int$ that $u \equiv 0$ is a

where $\lambda_{1}>0$ is (24) we conclud is 0 , and its loce

Let $g_{+}(x)=$ are the solution points of $\nabla J_{+}$ open set contain Schauder degre solutions is -1 . $\nabla J$ restricted tc

Following th changes sign exi then the local I the set of critica of $\nabla J$ is large b solution that $\mathrm{ch}$

where 1 accour Schauder degre of negative solu Leray-Schauder must have a ser of the proof of

If $j=k+1$ [21]). Also if $j$ exactly two solt

Thus it rem in terms of $\mid k-\jmath$ then number of for the hyperbo is a rational $\mathrm{mi}$ are needed due

\section{5. $\mathrm{Re}$}

In the hypo

$$
\frac{\partial f}{\partial u}(x,-c
$$


f $-\Delta$ or the wave eorem 4.1 applies ie following.

(2) or (16). If $f$ $y, u)$, and $[a, b] \cap$ ution.

to (16). In fact, rator defined by npact while that (16), is not so. hed.

with $\lim _{|u| \rightarrow \infty} h(x, u)$ then the problem solutions to (16) [58].

4.4 need not be er the hypotheses $y$ open questions.

u) $\in\left(\lambda_{j}, \lambda_{j+1}\right)$,

interval in $\mathbf{R}$, it $j \mid+1$ solutions. $[31]$, N. Dancer $-j \mid=N-1$ and if $k \neq j$ then (2) f $[\mathbf{2 1}],[\mathbf{1 6}]$, and

$j \geq 2$, and that hat $g^{\prime}(r) \leq$ a for solutions change where $G(t)=\int_{0}^{t} g(s) d s$. Since, trivially, $u \equiv 0$ is a solution to (22) we see that $u \equiv 0$ is a critical point of $J$. From (14) we see that

$$
\begin{aligned}
\left\langle D^{2} J(0) v, v\right\rangle & =\int_{\Omega}\left[\|\nabla v(x)\|^{2}-g^{\prime}(0) v^{2}\right] d x \\
& \geq\left(1-\frac{g^{\prime}(0)}{\lambda_{1}}\right) \int_{\Omega}\|\nabla v(x)\|^{2} d x,
\end{aligned}
$$

where $\lambda_{1}>0$ is as in (8) for the boundary condition $u=0$ on $\partial \Omega$. From (24) we conclude that $u \equiv 0$ is a local minimum. Moreover, its Morse index is 0 , and its local degree is +1 .

Let $g_{+}(x)=\max \{g(x), 0\}$. Let $J_{+}$be the functional whose critical points are the solutions to (22) when $g$ is replaced by $g_{+}$. Now the set of critical points of $\nabla J_{+}$is bounded and the Leray-Schauder degree of $\nabla J_{+}$on any open set containing all its critical points is equal to zero. Hence the LeraySchauder degree of $\nabla J$ restricted to any open set containing all positive solutions is -1 . Similar argument shows that the Leray-Schauder degree of $\nabla J$ restricted to any open set containing all negative solutions to (22) is -1 .

Following the arguments of $[\mathbf{1 6}]$ one sees that (22) has a solution $w$ that changes sign exactly once. Moreover such a solution is an isolated solution, then the local Leray-Schauder degree if $\nabla J$ is +1 . From (21) one sees that the set of critical points of $J$ is bounded and that the Leray-Schauder degree of $\nabla J$ is large balls centered at 0 is $(-1)^{j}$. Assuming that $(22)$ has one only solution that changes sign we have then that

$$
(-1)^{j}=1+(-1)+(-1)+1 \text {, }
$$

where 1 accounts for the local Leray-Schauder of $0,(-1)$ for the LearySchauder degree of the set of positive solutions to (22), similarly the set of negative solutions to (22), and the last term in (25) accounts for the local Leray-Schauder degree of $w$. Since (25) is contradictory we see that (22) must have a second solution that changes sign. This is concludes a sketch of the proof of Theorem 4.5 .

If $j=k+1$ and $r g^{\prime \prime}(r) \geq 0$, then (22) has exactly three solutions (see [21]). Also if $j=2$ then, under the assumptions of Theorem 4.5, (22) has exactly two solutions that change sign (see [15]).

Thus it remains open determining the number of solutions (2) must have in terms of $|k-j|$. It is tempting to conjecture that as $|k-j|$ tends to infinity, then number of solutions must tend to infinity. For a version Theorem 4.5 for the hyperbolic problem (16) the reader is referred to [8]. Even when $T$ is a rational multiple of $\pi$ additional hypotheses on the monotonicity of $f$ are needed due to the infinite multiplicity of the eigenvalue 0 .

\section{Resonance and the Landesman-Lazer condition}

In the hypotheses of the previous section the terms

$$
\frac{\partial f}{\partial u}(x,-\infty) \equiv \lim _{u \rightarrow-\infty} \frac{\partial f}{\partial u}(x, u), \text { and } \frac{\partial f}{\partial u}(x,+\infty) \equiv \lim _{u \rightarrow+\infty} \frac{\partial f}{\partial u}(x, u)
$$


stay in an interval contained in $\rho(L)$. Thus we can take advantage of the invertibility of $L-c I$. In contrast, in [38] the authors considered the case

$$
f(x, u)=\lambda_{k} u+g(u)+p(x),
$$

with $g$ bounded and monotone. For the sake of simplicity let us assume that $g$ is monotonically increasing. Let $g(-\infty) \equiv \lim _{u \rightarrow-\infty} g(u)<\lim _{u \rightarrow+\infty} g(u) \equiv$ $g(+\infty)$. When $(27)$ holds we say that the nonlinearity is in resonance with the eigenvalue $\lambda_{k}$. Surprisingly the following was established in [38].

THEOREM 5.1. Suppose $\lambda_{k}$ is a simple eigenvalue of $-\Delta$ subject to the Dirichlet boundary condition (see (7). Let $\phi_{k}$ be an eigenfunction associated with the eigenvalue $\lambda_{k}$. Let $\phi_{+}, \phi_{-}$be the positive and negative parts of $\phi_{k}$, respectively. If

$$
\begin{aligned}
g(-\infty) \int_{\Omega} \phi_{+}(x) d x & +g(+\infty) \int_{\Omega} \phi_{-}(x) d x<\int_{\Omega} p(x) \phi_{k}(x) d x \\
& <g(-\infty) \int_{\Omega} \phi_{-}(x) d x+g(+\infty) \int_{\Omega} \phi_{+}(x) d x,
\end{aligned}
$$

then (2) has a solution. Moreover, if $g(-\infty)<g(u)<g(+\infty)$ for all $u \in \mathbf{R}$, then (28) is a necessary condition for the solvability of (2).

Theorem 5.1 not only pioneers the solvability of nonlinear problems with non invertible linear part $\left(L=-\Delta+\lambda_{k} I\right)$, but also motivates important developments in variational methods. In fact, the saddle point principle proved by $\mathrm{P}$. Rabinowitz in [52] owes its origin to a variational interpretation of the hypothesis (28), also known as Landesman-Lazer condition.

Let us state a simplified version of the saddle point principle. Let $X, Y$ of $H$ such that $H=X \oplus Y$, and $\operatorname{dim} X<\infty$. Suppose that $\lim _{\|x\| \rightarrow \infty, x \in X} I(x)=$ $-\infty, \lim _{\|y\| \rightarrow \infty, y \in Y} I(y)=\infty$, and $I$ is bounded below on $Y$. Let $r>0$ be so large that

$$
\begin{aligned}
& \max \{I(x) ; x \in X,\|x\|=r\}<\inf \{I(y) ; y \in Y\}, \text { and } \\
& \quad \inf \{I(y) ; y \in Y,\|y\|=r\}>\max \{I(x) ;\|x\|=r, x \in X\} .
\end{aligned}
$$

THEOREM 5.2. Let $H$ be a Hilbert space and $I: H \rightarrow \mathbf{R}$ a functional of class $C^{1}$ and $r$ be as in (29). If

(PS) every sequence $\left\{u_{j}\right\}$ in $H$ such that $\left\{I\left(u_{j}\right)\right\}$ is bounded and $\lim _{j \rightarrow \infty} \nabla I\left(u_{j}\right)=0$ has a converging subsequence,

then

$$
c=\inf _{x \in \Sigma} \max _{\|x\|=r} I(s(x)),
$$

is a critical value, where $\Sigma=\{s:\{x \in X ;\|x\| \leq r\} \rightarrow H ; s$ is continuous, and $s(x)=x$ for $\|x\|=r\}$.

To derive Theorem 5.1 from the Saddle Point Principle we let $X$ and $Y$ be as in (15). From the first inequality in (15) it is easily seen that for $u \in X_{1}$ Similarly, $J(\imath$ $Y$. Thus app known as $\mathrm{Pal}$ assumption imply that $J$ reader is refe

For exten methods, and lems the read

The term refer to cases

In order to $\mathrm{s}$ nonlineariti

$$
-\infty<
$$

We recall tha sponding to we denote by $\phi_{1}(x)>0$ for and G. Prodi

THEOREM for each $q \in L$ that

has no solutic two solutions

This result ities. Hypothe assume that $u$ by $\phi_{1}$ and int $\epsilon$ $\lambda_{1} \int_{s}$ 
where $M$ is a lower bound for $g(u)-\lambda_{1} u$. Hence only if $c \geq M|\Omega|$ may (33) have a solution. This explains the nonexistence of solutions in Theorem 6.1. That (32) can have at most two solutions is strongly related to the nodal properties of eigenfunctions corresponding to the eigenvalue $\lambda_{2}$ (see Lemma 2.1).

If we replace in (32) the assumption $g^{\prime}(+\infty) \in\left(\lambda_{1}, \lambda_{2}\right)$ by $g^{\prime}(+\infty) \in$ $\left(\lambda_{k}, \lambda_{k+1}\right)$, one is tempted to conjecture that for large $c>0$ the equation (33) has $2 k$ solutior.s. This conjecture turns out to be correct when $\Omega$ is one dimensional, and even when $\Omega$ is a ball in $\mathbf{R}^{N}$ and one considers only radial solutions (see [17]) and [28]). E. N. Dancer in [33] provides counter examples to this general conjecture. To date, in general, all we can claim is that if $g^{\prime}(-\infty)<\lambda_{1}<\lambda_{k}<g^{\prime}(+\infty)<\lambda_{k+1}$ with $k \geq 2$, then for $c>0$ the problem (33) has at least four solutions. For early results on jumping nonlinearities the reader is referred to $[31],[40],[41],[42],[36]$, among others. For a survey on problems with jumping nonlinearities and their applications to the design of suspension bridges see [43]. For recent results in problems with jumping nonlinearities see [34], [50], and [56].

In $[\mathbf{3 6}], \mathrm{S}$. Fucik noticed the relevance of understanding the set

$$
\left\{(a, b) \text {; for some } u \in H-\{0\},-\Delta u=a u_{+} b u_{-}\right\}
$$

in the solvability of (33). This set is known as the Fucik spectrum of $-\Delta$ in $\Omega$. For additional information on the Fucik spectrum see [31] and [56]. Full understanding of the Fucik spectrum remains open. For the one dimensional case, a full characterization of the Fucik spectrum may be found in [36] for the case of Dirichlet boundary condition or periodic solutions.

After thirty years of Theorem 6.1 there remains the conjecture that, for $c>0$ large, the number of solutions to (33) is large when $k$ is large and $\lambda_{k}<g^{\prime}(+\infty)<\lambda_{k+1}$.

\section{Superlinear problems}

In this section we consider the superlinear case. That is when

$$
\lim _{|u| \rightarrow \infty} \frac{\partial f}{\partial u}(x, u)=\infty .
$$

This occurs, for example, if $f(x, u) \equiv g(u)=|u|^{\alpha} u$ and $\alpha>0$. Because the Sobolev space $H$ considered in Section 3 is not always contained in $L^{\infty}(\Omega)$, the functional $J$ need not be well defined in $H$. However, since $H$ is imbedded in $L^{p}(\Omega)$ for any $p \in\left(1,2^{*}\right)$ with $2^{*}=(N+2) /(N-2)$ for $N>2$, and $2^{*}=\infty$ for $N \leq 2$ (see $[\mathbf{1}]$ ), for $J$ to be well defined it is sufficient that

$$
|f(x, u)| \leq A\left(|u|^{p}+1\right),
$$

for some $p \in\left(1,2^{*}-1\right)$. When (37) is satisfied we say that $f$ has subcritical growth, or simply that $f$ is subcritical. Similarly we will say that $f$ is supercritical if there exist $p>2^{*}$ and $A>0$ such that

$$
|f(x, u)| \geq A\left(|u|^{p}-1\right) \text { for all }(x, u) \in \Omega \times \mathbf{R} .
$$

Finally we will

This guara is odd in the si to the Liuster solutions if, in

This condition the Palais-Sm also has infinit first variable the variable $u$, shown that (2 these structure solutions may general $f^{\prime} s$ pro problems has and P. L. Lior a perturbation $f(x, u)=|u|^{p-}$

where $H(x, t)$ if $N \leq 2), r$ $\beta^{\prime}<2 N /(N-$

THEOREM $\alpha)) /(N-2)$,

One notes $|u|^{\beta} u+p(x)$ fo it apply if $h \equiv$ some of the cas when $f$ is subc

In [51], S. 
$M|\Omega|$ may (33) in Theorem 6.1. ted to the nodal e $\lambda_{2}$ (see Lemma

2) by $g^{\prime}(+\infty) \in$ $>0$ the equation orrect when $\Omega$ is le considers only provides counter all we can claim 2, then for $c>0$ sults on jumping 12], [36], among arities and their or recent results $[56]$.

o the set

-

ectrum of $-\Delta$ in $1]$ and $[56]$. Full one dimensional found in $[36]$ for bns.

ajecture that, for $n k$ is large and

is when

$\alpha>0$. Because ays contained in wever, since $H$ is $V-2$ ) for $N>2$, is sufficient that

at $f$ has subcritvill say that $f$ is
Finally we will say that $f$ has critical growth if

$$
\lim _{|u| \rightarrow \infty} \frac{|f(x, u)|}{|u|^{2^{*}}} \in(0,+\infty) .
$$

This guarantees that the functional $J$ is of class $C^{1}$ (see [53]). When $f$ is odd in the second variable, $f(x, u)=-f(x,-u)$, arguments that go back to the Liusternik-Schnirelmann theory show that (2) has infinitely many solutions if, in addition, there exist $\theta>2$ and $\tau>0$ such that

$$
u f(x, u) \geq \theta F(x, u) \text { for }|u| \geq \tau, x \in \Omega .
$$

This condition plays a central role in proving that the functional $J$ satisfies the Palais-Smale condition. For $f$ not necessarily odd and $N=1$, (2) also has infinitely many solutions. If $\Omega$ is a ball in $\mathbf{R}^{N}, f$ is radial in the first variable $(f(x, u)=f(y, u)$ for $\|x\|=\|y\|)$ but not necessarily odd in the variable $u$, under additional structure conditions on $f$ it may also be shown that (2) has infinitely many solutions (see [18]). For the role of these structure conditions see Theorem 7.1 below. In fact, infinitely many solutions may be found even when $f$ exceeds the growth condition (37). For general $f^{\prime} s$ proving the existence of infinitely many solutions for superlinear problems has remained elusive. The following theorem, due to A. Bahri and P. L. Lions (see [5]), obtains existence of solutions to (2) when $f$ is a perturbation of $f_{1}(u) \equiv|u|^{p-1} u$ with $p \in\left(1,2^{*}\right)$. More precisely, let $f(x, u)=|u|^{p-1} u-h(x, u)$ with

$$
\begin{aligned}
&|h(x, t)| \leq p(x)+C|t|^{q} \text { for some } C \geq 0, p \in L_{+}^{r}(\Omega) \\
&|H(x, t)| \leq a(x)+b(x)|t|^{\alpha} \text { for some } \alpha \in(0,2),
\end{aligned}
$$

where $H(x, t)=\int_{0}^{t} h(x, s) d s, a \in L_{+}^{1}(\Omega), q=(N+2) /(N-2)(q<\infty$ if $N \leq 2), r=2 N /(N+2)(r>1$ if $N \leq 2), b \in L_{+}^{\beta}(\Omega)$ with $\beta>1$, $\beta^{\prime}<2 N /(N-2)(1 / \alpha)(\beta>1$ if $N \leq 2)$.

THEOREM 7.1. Let $f(x, u)=|u|^{p-1} u+h(x, u)$. If $p \in(1,(N+2(1-$ $\alpha)) /(N-2)$, then (2) has infinitely many solutions.

One notes that, for example, if $f(x, u)=|u|^{\alpha} u+p(x)$ for $u \geq 0, f(u)=$ $|u|^{\beta} u+p(x)$ for $u \leq 0$ and $\alpha \neq \beta$ Theorem 7.1 does not apply. Neither does it apply if $h \equiv 0$ and $p \in(N /(N-2),(N+2) /(N-2))$. These are just some of the cases that remain open in the case of superlinear problems, even when $f$ is subcritical and (40) holds.

\section{Critical exponents}

In [51], S. Pohozaev noted that if $u$ is a solution to

$$
\left\{\begin{array}{rlrl}
-\Delta u & =g(u) & & x \in \Omega \\
u(x) & =0 & x \in \partial \Omega,
\end{array}\right.
$$


then

$$
\frac{1}{2} \int_{\partial \Omega}\|\nabla u\|^{2}(x \cdot \nu) d s=N \int_{\Omega} G(u) d x-\frac{N-2}{2} \int_{\Omega} u g(u) d x .
$$

In the latter equations $\nu$ denotes the outward unit normal, and $d s$ the volume element on $\partial \Omega$. This identity, known as Pohozaev's identity, is particularly useful. For example, when $\Omega$ is a starlike region with respect to 0 the quantity $x \cdot \nu$ is positive, which forces $2 N G(t)-(N-2) \operatorname{tg}(t)$ to be nonnegative somewhere on the range of any solution $u$. Since, for example, $2 N G(t)-$ $(N-2) \operatorname{tg}(t)<0$ for $t \neq 0$ when $g(t)=|t|^{p-1} t$ with $p>2^{*}$, we see that for such $g$ 's the equation (42) has no nontrivial solution. With the help of the maximum principle on can also see that (42) has no nontrivial solution on starlike regions when $p=2^{*}$. This is in contrast with the case $p \in\left(1,2^{*}\right)$ where the problem has infinitely many solutions.

Despite the negative result of [51], in [9] H. Brezis and L. Nirenberg discovered the existence of solutions to (42) when $g(t)=\lambda t+|u|^{2^{*}-1} u$, and $N \geq 4$. In fact they proved that if $\lambda \in\left(0, \lambda_{1}\right)$, the problem (42) has a positive solution. These results in turn led for further understanding of the solvability of semilinear equations with critical growth (see (39)). The reader is referred to $[\mathbf{7}],[\mathbf{1 9}],[\mathbf{2 0}]$, and $[\mathbf{2 4}]$ for further developments in this respect. It must be noted that most of these results pertain to radially symmetric solutions. For general bounded regions the solvability of (42) is yet to be adequately explored.

\section{Qualitative properties of solutions}

While the above developments only state the existence, or multiplicity of solutions to semilinear problems, the methods of proof carry information on the nature of the solutions. For example when $f$ is nonnegative, owing to sub-supersolution methods one may determine that solutions to (2) are actually positive functions. The reader is referred to [45] for extensive information on this topic. Positive solutions may also exist when $f(x, 0)<0$ and $f(x, u)$ is positive for $u>0$ large. Equations of this type are known as semipositone equations (see [23]).

The study of solutions that change sign is relatively new. Motivated by the results of $[\mathbf{5 7}]$ the following was established in $[\mathbf{1 4}]$.

THEOREM 9.1. If $g(0)=0, g^{\prime}(0)<\lambda_{1}, t g^{\prime}(t)>g(t)$ for $t \neq 0$, g satisfies (40), and for some $p \in(1,(N+2) /(N-2), A \in(0, \infty)$

$$
\left|g^{\prime}(t)\right| \leq A\left(|t|^{p-1}+1\right) \text { for all } t \in \mathbf{R},
$$

then (42) has a solutions that changes sign exactly once (that is, $\{x \in$ $\Omega ; u(x)>0\}$ is connected and $\{x \in \Omega ; u(x)<0\}$ is connected.)

The existence of the solution in this theorem includes a variational characterization. Such a characterization has been skillfully used by J. M. Neuberger (see $[\mathbf{4 7}]$ and $[\mathbf{2 7}]$ ) to approximate such solution. The existence of 
$u g(u) d x$.

and $d s$ the volume ty, is particularly respect to 0 the to be nonnegative ample, $2 N G(t)-$ *, we see that for h the help of the rivial solution on e case $p \in\left(1,2^{*}\right)$

and L. Nirenberg $t+|u|^{2^{*}-1} u$, and blem (42) has a understanding of (see (39)). The developments in ertain to radially vability of (42) is

e, or multiplicity arry information nnegative, owing ations to (2) are for extensive inwhen $f(x, 0)<0$ pe are known as

w. Motivated by $t \neq 0, g$ satisfies (that is, $\{x \in$ ed.)

variational chard by J. M. NeuThe existence of solution that changes sign has also been used to establish the existence of other sign changing solutions (see [16]) and exact number of solutions that change sign (see [15]). For additional results in this direction, the reader is referred to $[6]$ and $[24]$.

While the assumption that $g$ grow subcritically is essential in the proof of Theorem [14], results for regions where $g$ grows at the critical rate have been recently found for regions with some symmetry (see [12] and [37]).

In case $g$ grows subcritically, say satisfying the assumption of Theorem 7.1, and $\Omega$ is a ball one may conclude the existence of infinitely many solutions either from the variational arguments of Theorem 7.1 or from onedimensional arguments as in [18]. In [2] it is shown that infinitely many of the solutions provided by Theorem 7.1 are nonradial. The proof is based on the analysis of the growth rate of energy levels of radial solutions that mimics the asymptotic expansion of radial versus nonradial eigenvalues.

Motivated by equations coming from, among others, biological models, extensive work has been devoted to finding nonnegative solutions to the Neumann problem (see [48])

$$
\left\{\begin{array}{lrl}
-\epsilon^{2} \Delta u & =g(u) & \\
\frac{\partial u}{\partial \eta}(x) & =0 & x \in \partial \Omega .
\end{array}\right.
$$

Here a key issue is the number and location of spikes of the solutions, and their behavior as $\epsilon$ tends to zero.

\section{Extensions and Applications}

The above results beg for extensions to cases where, for example, $L$ is non-selfadjoint, $L$ is quasilinear, or $L$ is given by a system of linear operators. Little is known when the operator $-\Delta$ is replaced in the above by a second order non-selfadjoint operator. Since most of the results have been derived not from the potential $J$ but rather from the vector field generated by the operator $u \rightarrow-\Delta u-f(x, u)$ on $H$, new ideas are needed to deal with the nonselfadjoint case. At this point one finds no reason to believe that, for example, if $-\Delta$ is perturbed by a first order operator then the above theorems extend to the new equation. That (5) has no nontrivial solution suggests, for example, that Theorem 7.1 does not extend to nonselfadjoint problems. For extensions to quasilinear problems the reader is referred to $[\mathbf{3 5}]$ and $[\mathbf{5 4}]$ and references therein.

The equation (1) models multiple phenomena. In fact the motivating examples may be derived from physical examples that go back to classical mathematical physics. For more recent applicability of semilinear models, the reader is referred to $[25],[43]$, and [49].

\section{References}

[1] R. Adams, Sobolev Spaces, New York, Academic Press (1975). 
[2] H. Aduen and A. Castro, Infinitely many nonradial solutions to a superlinear Dirichlet Prob-lem, Proc. Amer. Math. Soc. 131 (2003), pp. 835-843.

[3] A. Ambrosetti and G. Prodi, On the inversion of some differentiable mappings with singularities between Banach spaces, Ann. Mat. Pura Appl. 93(1972), 231-247.

[4] F. Atkinson, H. Brezis and L. Peletier, Solutions d'équations elliptiques avec exposant de Sobolev critique que changent de signe, C. R. Acad. Sci. Paris Serie I, (1988), vol.306, pp. 711-714.

[5] A. Bahri and P. L. Lions, Morse index of some min-max critical points. I. Application to multiplicity results, Comm. Pure Appl. Math. 41 (1988), no. 8, pp. 1027-1037.

[6] T. Bartsch, K.-C. Chang and Z.-Q. Wang On the Morse indices of sign changing solutions of nonlinear elliptic problems, Math. Z. 233 (2000), pp. 655-677.

[7] R. Benguria, J. Dolbeault, and M. Esteban, Classification of the solutions of semilinear elliptic problems in a ball, J. Differential Equations 167 (2000), no. 2, pp. 438-466.

[8] H. Brezis and M. Coron, Periodic solutions of nonlinear wave equations and Hamiltonian systems, Amer. J. Math. 103 (1981), no. 3, pp. 559-570.

[9] H. Brezis and L Nirenberg, Positive solutions of nonlinear elliptic equations involving critical Sobolev exponents, Comm. Pure Appl. Math. 36 (1983), no. 4, pp. 437-477.

[10] J. Caicedo and A. Castro, A semilinear wave equation with derivative of nonlinearity containing multiple eigenvalues of infinite multiplicity, . Contemp. Math., 208, pp. 111-132.

[11] A. Castro, Hammerstein integral equations with indefinite kernel, Internat. J. Math. Math. Sci. 1 (1978), no. 2, pp. 187-201.

[12] A. Castro and M. Clapp, The effect of the domain topology on the number of minimal nodal solutions of an elliptic equation at critical growth in a symmetric domain, to appear in Nonlinearity.

[13] A. Castro and J Cossio, Multiple solutions for a nonlinear Dirichlet problem, SIAM J. Math. Anal. 25 (1994), no. 6, pp. 1554-1561.

[14] A. Castro, J. Cossio, and J. M. Neuberger, A sign-changing solution for a superlinear Dirichlet problem, Rocky Mountain J. Math. 27 (1997), no. 4, pp. 1041-1053.

[15] A. Castro, J. Cossio, and J. M. Neuberger, On multiple solutions of a nonlinear Dirichlet problem, Nonlinear Anal. 30 (1997), no. 6, pp. 3657-3662.

[16] A. Castro, J. Cossio, and J. M. Neuberger, A minmax principle, index of the critical point, and existence of sign-changing solutions to elliptic boundary value problems, Electron. J. Differential Equations 1998, No. 02, 18 pp. (electronic).

[17] A. Castro and S. Gadam, The Lazer Mckenna Conjecture for Radial Solutions in the $\mathbf{R}^{N}$ Ball, Electron. J. Diff. Eqns. Vol. 1993(1993), No. 07, pp. 1-6.

[18] A. Castro and A. Kurepa, Infinitely many solutions to a superlinear Dirichlet problem in a ball, Proc. Amer. Math. Soc., (1987), vol. 101 No. 1, pp. 57-64.

[19] A. Castro and A. Kurepa, Radially symmetric solutions to a superlinear Dirichlet problem with jumping nonlinearities, Trans. Amer, Math. Soc., (1989), vol. 315, pp. 353-372

[20] A. Castro and A. Kurepa, Radially symmetric solutions to a Dirichlet problem involving critical exponents, Trans. Amer. Math. Soc., 348 (1996), no. 2, pp. 781-798.

[21] A. Castro and A. C. Lazer, Critical point theory and the number of solutions of a nonlinear Dirichlet problem, Ann. Mat. Pura Appl. (4) 120 (1979), pp. 113-137.

[22] A. Castro and S. Unsurangsie, A semilinear wave equation with nonmonotone nonlinearity, Pacific J. Math. 132 (1988), no, 2, pp. 215-225.

[23] A. Castro, C. Maya, and R. Shivaji, Nonlinear eigenvalue problems with semipositone structure, Electron. J. Differ. Equ., Conf. 5, (2000), pp. 33-49 (electronic).

[24] G. Cerami, S. Solimini, M. Struwe, Some existence results for superlinear elliptic boundary value problems involving critical exponents, J. Funct, Anal. 69 (1986), 289306.

[25] G. Chen, Z equation, ( $\mathrm{p}$

[26] S. N. Chow Berlin, (198

[27] D. Costa, Z changing so Appl. Math

[28] D. G. Coste ball, J. Diff,

[29] R. Courant John Wiley

[30] M. Crandal 8(1971), 32

[31] Dancer, E. equations, amb-prodi.

[32] E. N. Dance T.M.A., 13

[33] E. N. Dane nonlinear $e$

[34] E. N. Danc ear Anal. 1

[35] P. Drabek, theorem, J

[36] S. Fucik, B 101 (1976),

37] P. Girao, a growth in 407-419.

[38] E. Landesn value probl

[39] D. Kinderl Application

[40] A. C. Laze] problem, J. solutions w

[41] A. C. Laze problems \& Math. 368

[42] A. C. Laze with nonlin tions 11 (1 and $4-\mathrm{j}$ soli

[43] A. C. Laze bridges: so pp. $537-57$ ?

[44] A. C. Laze indices of , 775 .

[45] P. L. Lion SIAM Rev 
superlinear Dirichlet

atiable mappings with 1972), 231-247.

ptiques avec exposant Paris Serie I, (1988),

points. I. Application 8, pp. $1027-1037$. ces of sign changing . $655-677$.

he solutions of semi7 (2000), no. 2 , pp.

quations and Hamilc equations involving , no. 4, pp. 437-477. vative of nonlinearity mp. Math., 208, pp.

, Internat. J. Math.

e number of minimal mmetric domain, to

chlet problem, SLAM

ion for a superlinear p. 1041-1053.

tions of a nonlinear 62.

index of the critical lary value problems, iic).

dial Solutions in the 6.

ar Dirichlet problem 64.

uperlinear Dirichlet (1989), vol. 315, pp.

irichlet problem inno. 2 , pp. 781-798.

er of solutions of $a$ 9), pp. 113-137.

nonmonotone non-

is with semipositone ectronic).

superlinear elliptic nal. 69 (1986), 289-
[25] G. Chen, Z, Ding, C. Hu, W. Ni, and J. Zhou A note on the elliptic sine-Gordon equation, (preprint).

[26] S. N. Chow and J. K. Hale, Methods of bifurcation theory Springer-Verlag, New YorkBerlin, (1982).

[27] D. Costa, Z. Ding, and J. M. Neuberger, John M. A numerical investigation of signchanging solutions to superlinear elliptic equations on symmetric domains, J. Comput. Appl. Math. 131 (2001), no. 1-2, pp. 299-319.

[28] D. G. Costa and D. G. de Figueiredo, Radial solutions for a Dirichlet problem in a ball, J. Diff. Equations 60 (1985), pp. 80-89.

[29] R. Courant and D. Hilbert, Methods of Mathematical Physics, Volume I, New York, John Wiley (1989).

[30] M. Crandall and P. Rabinowitz, Bifurcation from simple eigenvalues, J. Funct. Anal. 8(1971), 321-340.

[31] Dancer, E. N. On the ranges of certain weakly nonlinear elliptic partial differential equations, J. Math. Pures Appl. (9) 57 (1978), no. 4, 351-366. (Abstract version of amb-prodi.)

[32] E. N. Dancer, A counter example to the Lazer-McKenna conjecture, Nonlinear Anal., T.M.A., 13(1989), 19-22.

[33] E. N. Dancer, Counterexamples to some conjectures on the number of solutions of nonlinear equations. Math. Ann., 272 (1985), no. 3, pp. 421-440.

[34] E. N. Dancer, Some results for jumping nonlinearities, Topological Methods Nonlinear Anal. 19 (2002), no. 2, 221-235. (35J60 (35J65 47Jxx 58E05).)

[35] P. Drabek, and S. Robinson, On the generalization of the Courant nodal domain theorem, J. Differential Equations 181 (2002), no. 1, pp. 58-71.

[36] S. Fucik, Boundary value problems with jumping nonlinearities, Casopis Pest. Mat. 101 (1976), no. 1, pp. 69-87.

[37] P. Girao, and M. Ramos, Sign changing solutions for elliptic equations with critical growth in cylinder type domains, ESAIM Control Optim. Calc. Var. 7 (2002), pp. 407-419.

[38] E. Landesman and A. C. Lazer, Nonlinear perturbations of linear elliptic boundary value problems at resonance, J. Math. Mech. 19 1969/1970, pp. 609-623.

[39] D. Kinderlehrer-G. Stampacchia, Introduction to Variational Inequalities and Their Applications, New York, Academic Press (1979).

[40] A. C. Lazer and P. J. McKenna, On the number of solutions of a nonlinear Dirichlet problem, J. Math. Anal. Appl. 84 (1981), no. 1, 282-294. (Existence of at least four solutions when crossing from below the first eigenvalue to one larger than the second.)

[41] A. C. Lazer and P. J. McKenna, Multiple of solutions of nonlinear boundary value problems with nonlinearities crossing several higher eigenvalues, J. Reine Angew, Math. 368 (1986), 184-200. (existence of our solutions.)

[42] A. C. Lazer and P. J. McKenna, Critical point theory and boundary value problems with nonlinearities crossing multiple eigenvalues, II. Comm. Partial Differential Equations 11 (1986), no. 15, 1653-1676. (Existence of $\mathrm{j}$ solutions $(\mathrm{j}$ in $(1,3))$ for c large and 4 -j solutions for c large negative.)

[43] A. C. Lazer and P. J. McKenna, Large-amplitude periodic oscillations in suspension bridges: some new connections with nonlinear analysis. SIAM Rev. 32 (1990), no. 4, pp. $537-578$.

[44] A. C. Lazer and S. Solimini, Nontrivial solutions of operator equations and Morse indices of critical points of min-max type, Nonlinear Anal. 12 (1988), no, 8, pp. 761 775 .

[45] P. L. Lions, On the existence of positive solutions of semilinear elliptic equations, SIAM Rev. 24 (1982), no. 4, pp. 441-467. 
[46] P. J. McKenna, On solutions of a nonlinear wave equation when the ratio of the period to the length of the interval is irrational, Proc. Amer. Math. Soc. 93 (1985), no. 1, pp. $59-64$.

[47] J. M. Neuberger, A numerical method for finding sign-changing solutions of superlinear Dirichlet problems, Nonlinear World 4 (1997), no. 1, 73-83.

[48] W. Ni, I. Takagi, and J. Wei, On the location and profile of spike-layer solutions to a singularly perturbed semilinear Dirichlet problem: intermediate solutions, Duke Math. J. 94 (1998), no. 3, pp. 597-618.

[49] S. Oruganti, J. Shi, and R. Shivaji, Diffusive logistic equation with constant yield harvesting. I. Steady states, Trans. Amer. Math. Soc. 354 (2002), no. 9, pp. 36013619 (electronic.)

[50] K. Perera, Resonance problems with respect to the Fučik spectrum of the p-Laplacian, Electron. J. Differential Equations 2002, No. 36, 10 pp. (electronic.)

[51] S. I. Pohozaev, On the eigenfunctions of the equation $\Delta u+\lambda f(u)=0$. Dokl. Akad. Nauk SSSR 165 (965), pp. 36-39.

[52] P. H. Rabinowitz, Some minimax theorems and applications to nonlinear partial differential equations, Nonlinear analysis (collection of papers in honor of Erich $\mathrm{H}$. Rothe), Academic Press, New York, (1978), pp. 161-177.

[53] P. H. Rabinowitz, Minimax methods in critical point theory with applications to differential equations, CBMS Regional Conference Series in Mathematics, No. 65. Published for the Conference Board of the Mathematical Sciences, Washington, DC; and the American Mathematical Society, Providence, RI, (1986).

[54] V. Shapiro, Singular quasilinearity and higher eigenvalues, Mem. Amer. Math. Soc. 153 (2001), no. 726 .

[55] M. Schechter, Linking Methods in Critical Point Theory, Springer Verlag, New York, (1999),

[56] H. Therani, A multiplicity result for the jumping nonlinearity problem, preprint.

[57] Z. Q. Wang, On a Superlinear Elliptic Equation, Ann. Inst. H. Poincare Analyse Non Lineaire 8 (1991), 43-57.

[58] M. Willem, Variational methods and almost solvability of semilinear equations, Lecture Notes in Math., 846, Springer Verlag, Berlin, (1981).

E-mail address: Castro@math.hmc.edu 